\title{
Ranking Semantics for Argumentation Systems With Necessities
}

\author{
Dragan Doder $^{1 *}$, Srdjan Vesic $^{2}$ and Madalina Croitoru ${ }^{3}$ \\ ${ }^{1}$ Utrecht University, the Netherlands \\ ${ }^{2}$ CRIL - CNRS, Univ. Artois, France \\ ${ }^{3}$ University of Montpellier, France \\ d.doder@uu.nl, vesic@cril.fr, croitoru@lirmm.fr
}

\begin{abstract}
Bipolar argumentation studies argumentation graphs where attacks are combined with another relation between arguments. Many kind of relations (e.g. deductive support, evidential support, necessities etc.) have been defined and investigated from a Dung semantics perspective. We place ourselves in the context of argumentation systems with necessities and provide the first study to investigate ranking semantics in this setting. To this end, we (1) provide a set of postulates specifically designed for necessities and (2) propose the first ranking-based semantics in the literature to be shown to respect these postulates.
\end{abstract}

\section{Introduction}

Argumentation framework is a simple yet powerful knowledge representation and reasoning paradigm [Dung, 1995]. The underlying representation structure is a directed graph where the nodes represent arguments and the directed edges represent attacks between the arguments. Over this structure, two main classes of semantics were proposed for reasoning. Extension-based semantics rely on selecting subsets of arguments (called extensions) based on specific properties within the graph [Dung, 1995]. Ranking-based semantics provide an order between the arguments of the graph [Matt and Toni, 2008; Leite and Martins, 2011; Amgoud and BenNaim, 2013; Bonzon et al., 2016; Amgoud and Doder, 2018; Amgoud and Doder, 2019]. Roughly speaking, an intuitive difference between the two classes of semantics is that, in extension based semantics, the attack relation is mostly used to destroy its target (two arguments attacking each other will not be in the same extension) whereas in ranking based semantics it is often used to only weaken its target.

Bipolar argumentation frameworks are a generalization of argumentation graphs in which we consider as underlying representation a bi-colored graph: the nodes represent arguments and the bi-colored edges represent, respectively, support and attack relations between the arguments [Cayrol and Lagasquie-Schiex, 2005b; Amgoud et al., 2008; Boella et al., 2010; Cayrol and Lagasquie-Schiex, 2013].

\footnotetext{
${ }^{*}$ Contact Author
}

Bipolar argumentation systems have received much attention in the literature due to the versatility of their representation power. The various relations defined in complement to attacks can express several kinds of endorsements: deductive [Villata et al., 2012], evidential [Oren and Norman, 2008], necessities [Nouioua and Risch, 2011; Nouioua, 2013; Cohen et al., 2015; Gottifredi et al., 2018] etc.

In this paper we turn our attention to the study of ranking semantics over bipolar argumentation frameworks with necessities (i.e. argument A is necessary for argument B) that have been proposed in order to model knowledge such as "if argument B is accepted then necessarily argument A is accepted". Please note that these argumentation frameworks have only been investigated from an extension-based semantics point of view [Nouioua and Risch, 2011; Nouioua, 2013; Gottifredi et al., 2018].

To illustrate the significance of our contribution, let us consider a motivating example. In certain countries (France, Italy, Germany etc) a diploma of habilitation is required for the candidate in order to apply for a professorship. The selection is then based on the significance of the research and teaching activities. The necessity of having a habilitation cannot be discussed, it is a requirement. Thus, the argument of having the habilitation is not reinforcing the argument of being a professor, but, on the contrary, it can destroy it.

To illustrate the different nature of the two relations, observe the argumentation graph from Figure 1. Let argument $a$ be "Tom should be hired as a professor". Since the habilitation is a necessary condition, argument $b$, standing for "Tom has a habilitation diploma", is necessary for $a$. This is depicted by a dotted blue arrow from $b$ to $a$. Let $c$ stand for "Tom has only a few publications", and $d$ for "Tom does not have a lot of teaching experience". The facts that $c$ and $d$ attack $a$ are represented by the solid red arrows.

What we need to model in this example is the following: On one hand, each of the attacks from $c$ and $d$ weakens the argument $a$, but none of those attacks is sufficient to completely reject $a$, hence it is convenient to use a ranking-based semantics to model this behaviour. On the other hand, if $b$ is rejected, $a$ should be rejected as well. This necessity link can not be modeled by an attack relation in ranking based semantics. Namely, suppose that one introduces an argument $b^{\prime}$ with meaning that "Tom does not have a habilitation", and an attack from $b^{\prime}$ to $a$. This modeling choice is not appropriate, 


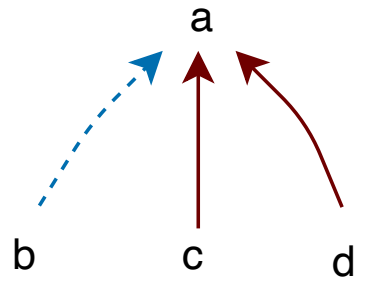

Figure 1: Bipolar argumentation graph with necessities.

since $b^{\prime}$ cannot completely destroy $a$.

Hence, to model this situation, we will use ranking based semantics with necessities. To this end, the contribution of this paper is two-fold as follows:

- A modelling contribution unravelling the particular role that the necessity relation plays in the context of ranking based semantics for bipolar argumentation frameworks.

- A technical contribution consisting on (1) a set of postulates specifically designed for describing ranking based semantics in the context of argumentation frameworks with necessities and (2) the first ranking based semantics in the literature for argumentation frameworks with necessities in argumentation frameworks without cycles.

The rest of the paper is organized as follows: Section 2 introduces the basic notions necessary for this work. In Section 3 we propose the set of principles for ranking based semantics for argumentation frameworks with necessities. Section 4 presents the formal analysis of the principles, studies the links between them and shows the impact of the principles to the behavior of the framework. In Section 5 we define the first gradual semantics for argumentation frameworks with necessities, we prove a characterization result and we verify that the introduced semantics satisfies all the principles. We conclude in Section 6.

\section{Formal Setting}

In this paper we follow the standard framework for weighted argumentation graphs [Amgoud et al., 2017], and extend it with a necessity relation between arguments. The resulting framework, that we named weighted argumentation graph with necessities is a graph that consists of a finite set of arguments and two binary relations between them: a necessity relation and an attack relation. In addition ${ }^{1}$, an initial weight from the unit interval of reals $[0,1]$ is attached to each argument.

Definition 1 (WGN) $A$ weighted argumentation graph with necessities (WGN) is a tuple $\mathbf{G}=\langle\mathcal{A}, w, \mathcal{R}, \mathcal{N}\rangle$, where

- $\mathcal{A}$ is a non-empty finite set of arguments,

- $w: \mathcal{A} \rightarrow[0,1]$

- $\mathcal{R} \subseteq \mathcal{A} \times \mathcal{A}$,

${ }^{1}$ Please note that, for generality reasons, we will consider weighted argumentation frameworks in the remainder of the paper.
- $\mathcal{N} \subseteq \mathcal{A} \times \mathcal{A}$, where $\mathcal{N}$ is acyclic, and

- $\mathcal{R} \cap \mathcal{N}=\emptyset$.

Intuitively, $w(a)$ is the initial strength of argument $a$, i.e. the intrinsic weight attached to it independently of the attacks between the arguments. For example, an argument coming from a more trustworthy source will be attached a higher initial weight. Notation $(a, b) \in \mathcal{R}$ (or $a \mathcal{R} b$ ) means that argument $a$ attacks argument $b$ and $(a, b) \in \mathcal{N}($ or $a \mathcal{N} b)$ means argument $a$ is necessary for argument $b . \operatorname{Att}_{\mathbf{G}}(a)$ denotes the set of all attackers of $a$ in $\mathbf{G}$, and $\operatorname{Nec}_{\mathbf{G}}(a)$ denotes the set of all arguments necessary for $a$ in $\mathbf{G}$. As in the previous work on argumentation frameworks with necessities [Gottifredi et al., 2018; Boudhar et al., 2012], we assume that $\mathcal{N}$ is acyclic and that $\mathcal{R} \cap \mathcal{N}=\emptyset$.

Definition 2 (Semantics) A semantics is a function $\mathbf{S}$ which maps any $W G N \mathbf{G}=\langle\mathcal{A}, w, \mathcal{R}, \mathcal{N}\rangle$ into a function $\operatorname{Deg}_{\mathbf{G}}^{\mathbf{S}}$ : $\mathcal{A} \rightarrow[0,1]$.

For any $a \in \mathcal{A}, \operatorname{Deg}_{\mathbf{G}}^{\mathbf{S}}(a)$ represents the overall strength of argument $a$ in the graph $\mathbf{G}$.

\section{Principles for Weighted Argumentation With Necessities}

In this section, we propose the first set of principles for ranking-based semantics for argumentation frameworks with necessities. Those principles can be categorized in three classes. Some of them are direct adaptations of the existing principles for weighted argumentation frameworks [Amgoud et al., 2017] (e.g. independence states that two disjoint graphs do not influence the degrees of each other's arguments). Other principles extend the existing principles (e.g. weakening soundness states that there are two reasons for the loss of strength of an argument, one of them being the necessity relation).

In addition, we propose five novel principles that describe the impact of necessity relation to the semantics: n-neutrality, n-weakening, n-counting, $n$-reinforcement and trimming. Some of them have counterparts amongst the principles for attack relations (e.g. n-reinforcement states that increasing the strength of a necessary argument increases the strength of its target, in contrast to a-reinforcement which states that increasing the strength of an attacker decreases the strength of the target), while some of them do not (e.g. trimming states that an argument cannot be more acceptable than any argument that is necessary for it).

We point out that a-reinforcement corresponds to the principe bi-variate reinforcement designed by Amgoud and Ben-Naim [2018] for the support relation. The other four novel principles are essentially different from the existing principles of Amgoud and Ben-Naim due to the different natures of necessity and support relations.

Let us first define the notion of isomorphism.

Definition 3 (Isomorphism) Let $\mathbf{G}=\langle\mathcal{A}, w, \mathcal{R}, \mathcal{N}\rangle$ and $\mathbf{G}^{\prime}=\left\langle\mathcal{A}^{\prime}, w^{\prime}, \mathcal{R}^{\prime}, \mathcal{N}^{\prime}\right\rangle$ be two WGNs. An isomorphism from $\mathbf{G}$ to $\mathbf{G}^{\prime}$ is a bijective function $f$ from $\mathcal{A}$ to $\mathcal{A}^{\prime}$ such that:

- $\forall a \in \mathcal{A}, w(a)=w^{\prime}(f(a))$,

- $\forall a, b \in \mathcal{A}, a \mathcal{R} b$ iff $f(a) \mathcal{R}^{\prime} f(b)$. 
- $\forall a, b \in \mathcal{A}, a \mathcal{N} b$ iff $f(a) \mathcal{N}^{\prime} f(b)$.

We can now formulate the anonymity principle.

Principle 1 (Anonymity) A semantics $\mathbf{S}$ satisfies anonymity iff, for any two WGNs $\mathbf{G}=\langle\mathcal{A}, w, \mathcal{R}, \mathcal{N}\rangle$ and $\mathbf{G}^{\prime}=$ $\left\langle\mathcal{A}^{\prime}, w^{\prime}, \mathcal{R}^{\prime}, \mathcal{N}^{\prime}\right\rangle$, for any isomorphism ff from $\mathbf{G}$ to $\mathbf{G}^{\prime}$, the following property holds: $\forall a \in \mathcal{A}$,

$$
\operatorname{Deg}_{\mathbf{G}}^{\mathbf{S}}(a)=\operatorname{Deg}_{\mathbf{G}^{\prime}}^{\mathbf{S}}(f(a)) .
$$

According to the independence principle, unconnected parts of a graph do not impact each other.

Principle 2 (Independence) A semantics $\mathbf{S}$ satisfies independence iff, for any two WGNs $\mathbf{G}=\langle\mathcal{A}, w, \mathcal{R}, \mathcal{N}\rangle$ and $\mathbf{G}^{\prime}=\left\langle\mathcal{A}^{\prime}, w^{\prime}, \mathcal{R}^{\prime}, \mathcal{N}^{\prime}\right\rangle$ s.t. $\mathcal{A} \cap \mathcal{A}^{\prime}=\emptyset$, the following holds: $\forall a \in \mathcal{A}, \operatorname{Deg}_{\mathbf{G}}^{\mathbf{S}}(a)=\operatorname{Deg}_{\mathbf{G} \oplus \mathbf{G}^{\prime}}^{\mathbf{S}}(a)$, where $\mathbf{G} \oplus \mathbf{G}^{\prime}=$ $\left\langle\mathcal{A} \cup \mathcal{A}^{\prime}, w^{\prime \prime}, \mathcal{R} \cup \mathcal{R}^{\prime}, \mathcal{N} \cup \mathcal{N}^{\prime}\right\rangle$ with $w^{\prime \prime}$ such that for every $a \in \mathcal{A}$ (resp. $a^{\prime} \in \mathcal{A}^{\prime}$ ) we have $w^{\prime \prime}(a)=w(a)$ (resp. $\left.w^{\prime \prime}\left(a^{\prime}\right)=w^{\prime}\left(a^{\prime}\right)\right)$.

A path from $a$ to $b$ in a WGN $\mathbf{G}=\langle\mathcal{A}, w, \mathcal{R}, \mathcal{N}\rangle$ is any sequence $a=a_{0}, a_{1}, \ldots, a_{n}=b$ of arguments such that $\left(a_{i-1}, a_{i}\right) \in \mathcal{R} \cup \mathcal{N}$ for every $i \in\{1, \ldots, n\}$.

Principle 3 (Directionality) A semantics $\mathbf{S}$ satisfies directionality iff, for any two WGNs $\mathbf{G}=\langle\mathcal{A}, w, \mathcal{R}, \mathcal{N}\rangle$ and $\mathbf{G}^{\prime}=\left\langle\mathcal{A}, w, \mathcal{R}^{\prime}, \mathcal{N}^{\prime}\right\rangle$ s.t. either

- $\mathcal{R}^{\prime}=\mathcal{R} \cup\{(a, b)\}$ and $\mathcal{N}=\mathcal{N}^{\prime}$, or

- $\mathcal{N}^{\prime}=\mathcal{N} \cup\{(a, b)\}$ and $\mathcal{R}=\mathcal{R}^{\prime}$,

for every $c \in \mathcal{A}$ such that there is no path from $b$ to $c$, we have $\operatorname{Deg}_{\mathbf{G}}^{\mathbf{S}}(c)=\operatorname{Deg}_{\mathbf{G}^{\prime}} \mathbf{S}^{\prime}(c)$.

According to the a-neutrality principle, the attackers of degree 0 do not impact their targets.

Principle 4 (A-neutrality) $A$ semantics $\mathbf{S}$ satisfies Aneutrality iff, for any $W G N \mathbf{G}=\langle\mathcal{A}, w, \mathcal{R}, \mathcal{N}\rangle, a, b \in \mathcal{A}$, if

- $w(a)=w(b)$,

- $\operatorname{Att}_{\mathrm{G}}(b)=\operatorname{Att}_{\mathrm{G}}(a) \cup\{c\}$ with $c \in \mathcal{A} \backslash \operatorname{Att}_{\mathrm{G}}(a)$, $\operatorname{Deg}_{\mathbf{G}}^{\mathbf{S}}(c)=0$, and

- $\operatorname{Nec}_{\mathbf{G}}(b)=\operatorname{Nec}_{\mathbf{G}}(a)$,

then $\operatorname{Deg}_{\mathbf{G}}^{\mathbf{S}}(a)=\operatorname{Deg}_{\mathbf{G}}^{\mathbf{S}}(b)$.

According to the $N$-neutrality principle, the necessary arguments of degree 1 do not impact their targets.

Principle 5 (N-neutrality) $A$ semantics $\mathbf{S}$ satisfies $\mathrm{N}$ neutrality iff, for any $W G N \mathbf{G}=\langle\mathcal{A}, w, \mathcal{R}, \mathcal{N}\rangle, a, b \in \mathcal{A}$, if

- $w(a)=w(b)$,

- $\operatorname{Att}_{\mathbf{G}}(b)=\operatorname{Att}_{\mathbf{G}}(a)$, and

- $\operatorname{Nec}_{\mathbf{G}}(b)=\operatorname{Nec}_{\mathbf{G}}(a) \cup\{c\}$ with $c \in \mathcal{A} \backslash \operatorname{Nec}_{\mathbf{G}}(a)$, $\operatorname{Deg}_{\mathbf{G}}^{\mathbf{S}}(c)=1$,

then $\operatorname{Deg}_{\mathbf{G}}^{\mathbf{S}}(a)=\operatorname{Deg}_{\mathbf{G}}^{\mathbf{S}}(b)$.

The equivalence principle states that two arguments having the attackers and the necessary arguments with the same degrees also have the same degrees, provided that their initial weights are same.
Principle 6 (Equivalence) $A$ semantics $\mathbf{S}$ satisfies equivalence iff, for any $W G N \mathbf{G}=\langle\mathcal{A}, w, \mathcal{R}, \mathcal{N}\rangle$ and $a, b \in \mathcal{A}$, if

- $w(a)=w(b)$

- there exists a bijection $f: \operatorname{Att}_{\mathrm{G}}(a) \rightarrow \operatorname{Att}_{\mathrm{G}}(b)$ such that $\operatorname{Deg}_{\mathbf{G}}^{\mathbf{S}}(c)=\operatorname{Deg}_{\mathbf{G}}^{\mathbf{S}}(f(c))$ for every $c \in \operatorname{Att}_{\mathbf{G}}(a)$,

- there exists a bijection $g: \operatorname{Nec}_{\mathrm{G}}(a) \rightarrow \operatorname{Nec}_{\mathrm{G}}(b)$ such that $\operatorname{Deg}_{\mathbf{G}}^{\mathbf{S}}(c)=\operatorname{Deg}_{\mathbf{G}}^{\mathbf{S}}(g(c))$ for every $c \in \operatorname{Nec}_{\mathbf{G}}(a)$,

then $\operatorname{Deg}_{\mathbf{G}}^{\mathbf{S}}(a)=\operatorname{Deg}_{\mathbf{G}}^{\mathbf{S}}(b)$.

According to the maximality principle, the degree of an argument which does not have any attackers nor necessary arguments is exactly its initial weight.

Principle 7 (Maximality) A semantics $\mathbf{S}$ satisfies maximality iff, for any $W G N \mathbf{G}=\langle\mathcal{A}, w, \mathcal{R}, \mathcal{N}\rangle$, and every $a \in \mathcal{A}$ such that $\operatorname{Att}_{\mathbf{G}}(a)=\operatorname{Nec}_{\mathbf{G}}(a)=\emptyset$, we have

$$
\operatorname{Deg}_{\mathbf{G}}^{\mathbf{S}}(a)=w(a) \text {. }
$$

The a-weakening principle states that any attack from an argument having a positive degree necessarily leads to the loss of the initial weight of the target.

Principle 8 (A-weakening) A semantics $\mathbf{S}$ satisfies aweakening iff, for any $W G N \mathbf{G}=\langle\mathcal{A}, w, \mathcal{R}, \mathcal{N}\rangle$, for any argument $a \in \mathcal{A}$, if

- $w(a)>0$, and

- there exists $b \in \operatorname{Att}_{\mathbf{G}}(a)$ such that $\operatorname{Deg}_{\mathbf{G}}^{\mathbf{S}}(b)>0$, then $\operatorname{Deg}_{\mathbf{G}}^{\mathbf{S}}(a)<w(a)$.

Recall that by n-neutrality, perfect necessary arguments (of degree 1) do not impact the target. On the contrary, if a necessary argument is imperfect (i.e has a degree strictly smaller than 1), that will lead to some loss of the initial weight of the target. This is formalized by the following principle.

Principle 9 (N-weakening) A semantics $\mathbf{S}$ satisfies $\mathrm{n}$ weakening iff, for any $W G N \mathbf{G}=\langle\mathcal{A}, w, \mathcal{R}, \mathcal{N}\rangle$, for any argument $a \in \mathcal{A}$, if

- $w(a)>0$, and

- there exists $b \in \operatorname{Nec}_{\mathbf{G}}(a)$ such that $\operatorname{Deg}_{\mathbf{G}}^{\mathbf{S}}(b)<1$, then $\operatorname{Deg}_{\mathbf{G}}^{\mathbf{S}}(a)<w(a)$.

The a-counting principle states that every attacker will decrease the degree of the target, provided that the attacker's acceptability degree is strictly positive.

Principle 10 (A-counting) $A$ semantics $\mathbf{S}$ satisfies acounting iff, for any $W G N \mathbf{G}=\langle\mathcal{A}, w, \mathcal{R}, \mathcal{N}\rangle$ and $a, b \in \mathcal{A}$, if

- $w(a)=w(b)$,

- $\operatorname{Deg}_{\mathbf{G}}^{\mathbf{S}}(a)>0$,

- $\operatorname{Nec}_{\mathrm{G}}(a)=\operatorname{Nec}_{\mathrm{G}}(b)$, and

- $\operatorname{Att}_{\mathbf{G}}(b)=\operatorname{Att}_{\mathbf{G}}(a) \cup\{c\}$ with $c \notin \operatorname{Att}_{\mathrm{G}}(a)$ and $\operatorname{Deg}_{\mathbf{G}}^{\mathbf{S}}(c)>0$,

then $\operatorname{Deg}_{\mathbf{G}}^{\mathbf{S}}(a)>\operatorname{Deg}_{\mathbf{G}}^{\mathbf{S}}(b)$. 
The next principle, called n-counting, formalizes the intuition that adding a necessary argument having a degree non equal to one will decrease the acceptability degree of the target (provided that the target does not already have the zero degree).

Principle 11 (N-counting) A semantics $\mathrm{S}$ satisfies $\mathrm{n}$ counting iff, for any $W G N \mathbf{G}=\langle\mathcal{A}, w, \mathcal{R}, \mathcal{N}\rangle$ and $a, b \in \mathcal{A}$, if

- $w(a)=w(b)$,

- $\operatorname{Deg}_{\mathbf{G}}^{\mathbf{S}}(a)>0$,

- $\operatorname{Att}_{\mathrm{G}}(a)=\operatorname{Att}_{\mathrm{G}}(b)$, and

- $\operatorname{Nec}_{\mathrm{G}}(b)=\operatorname{Nec}_{\mathrm{G}}(a) \cup\{c\}$ with $c \notin \operatorname{Nec}_{\mathrm{G}}(a)$ and $\operatorname{Deg}_{\mathbf{G}}^{\mathbf{S}}(c)<1$,

then $\operatorname{Deg}_{\mathbf{G}}^{\mathbf{S}}(a)>\operatorname{Deg}_{\mathbf{G}}^{\mathbf{S}}(b)$.

Weakening soundness identifies the only two possible reasons for losing some initial weight, namely, being attacked by non zero degree arguments and having necessary arguments with degree different from 1 .

Principle 12 (Weakening soundness) A semantics $\mathrm{S}$ satisfies weakening soundness iff, for any WGN $\mathbf{G}=$ $\langle\mathcal{A}, w, \mathcal{R}, \mathcal{N}\rangle$, and $a \in \mathcal{A}$, if $\operatorname{Deg}_{\mathbf{G}}^{\mathbf{S}}(a)<w(a)$, then there exists either $b \in \operatorname{Att}_{\mathbf{G}}(a)$ such that $\operatorname{Deg}_{\mathbf{G}} \mathbf{S}(b)>0$, or $b \in \operatorname{Nec}_{\mathbf{G}}(a)$ such that $\operatorname{Deg}_{\mathbf{G}} \mathbf{S}(b)<1$.

A-reinforcement states that a stronger attacker has a bigger impact on a target.

Principle 13 (A-reinforcement) A semantics $\mathbf{S}$ satisfies areinforcement iff, for any $W G N \mathbf{G}=\langle\mathcal{A}, w, \mathcal{R}, \mathcal{N}\rangle$, and $a, b \in \mathcal{A}$, if

- $w(a)=w(b)$,

- $\operatorname{Deg}_{\mathbf{G}}^{\mathbf{S}}(a)>0$ or $\operatorname{Deg}_{\mathbf{G}}^{\mathbf{S}}(b)>0$,

- $\operatorname{Nec}_{\mathrm{G}}(a)=\operatorname{Nec}_{\mathrm{G}}(b)$,

- $\operatorname{Att}_{\mathrm{G}}(a) \backslash \operatorname{Att}_{\mathrm{G}}(b)=\{x\}$,

- $\operatorname{Att}_{\mathbf{G}}(b) \backslash \operatorname{Att}_{\mathbf{G}}(a)=\{y\}$, and

- $\operatorname{Deg}_{\mathbf{G}}^{\mathbf{S}}(y)>\operatorname{Deg}_{\mathbf{G}}^{\mathbf{S}}(x)$,

then $\operatorname{Deg}_{\mathbf{G}}^{\mathbf{S}}(a)>\operatorname{Deg}_{\mathbf{G}}^{\mathbf{S}}(b)$.

On the contrary to a-reinforcement, n-reinforcement claims that a necessary argument with a stronger degree weakens its target less than a necessary argument with a weaker degree.

Principle 14 (N-reinforcement) A semantics $\mathbf{S}$ satisfies reinforcement iff, for any $W G N \mathbf{G}=\langle\mathcal{A}, w, \mathcal{R}, \mathcal{N}\rangle$, and $a, b \in$ $\mathcal{A}$, if

$$
\begin{aligned}
& \text { - } w(a)=w(b), \\
& \text { - } \operatorname{Deg}_{\mathbf{G}}^{\mathbf{S}}(a)>0 \text { or } \operatorname{Deg}_{\mathbf{G}}^{\mathbf{S}}(b)>0, \\
& \text { - } \operatorname{Att}_{\mathbf{G}}(a)=\operatorname{Att}_{\mathbf{G}}(b), \\
& \text { - } \operatorname{Nec}_{\mathbf{G}}(a) \backslash \operatorname{Nec}_{\mathbf{G}}(b)=\{x\}, \\
& \text { - } \operatorname{Nec}_{\mathbf{G}}(b) \backslash \operatorname{Nec}_{\mathbf{G}}(a)=\{y\}, \text { and } \\
& \text { - } \operatorname{Deg}_{\mathbf{G}}^{\mathbf{S}}(y)>\operatorname{Deg}_{\mathbf{G}}^{\mathbf{S}}(x), \\
& \text { then } \operatorname{Deg}_{\mathbf{G}}^{\mathbf{S}}(b)>\operatorname{Deg}_{\mathbf{G}}^{\mathbf{S}}(a) .
\end{aligned}
$$

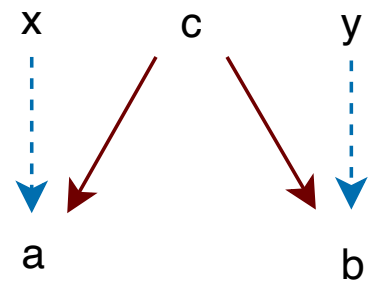

Figure 2: Graph $\mathbf{G}$ from Example 1.

Example 1 Let $\mathbf{G}$ be the WGN from Figure 2, where red solid arrows represent attacks and blue dotted arrow represent necessities. Let $w(a)=0.9, w(b)=0.9, w(c)=$ $0.5, w(x)=0.6$ and $w(y)=0.8$. Assume that semantics $\mathbf{S}$ satisfies maximality. Therefore, $\operatorname{Deg}_{\mathbf{G}}^{\mathbf{S}}(x)=0.6$ and $\operatorname{Deg}_{\mathbf{G}} \mathbf{S}(y)=0.8$. Since $\operatorname{Att}(a)=\operatorname{Att}(b)=\{c\}$ and $\operatorname{Deg}_{\mathbf{G}}^{\mathbf{S}}(x)<\operatorname{Deg}_{\mathbf{G}}^{\mathbf{S}}(y)$, by n-reinforcement we obtain that $\operatorname{Deg}_{\mathbf{G}}^{\mathbf{S}}(a)<\operatorname{Deg}_{\mathbf{G}}^{\mathbf{S}}(b)$.

The introduced principles emphasize that the necessity relation is essentially different from both attack and support relations. Indeed, according to n-weakening, necessities and attacks share a crucial property, which clearly distinguishes them from support relations - they both decrease the degrees of their targets. In addition, according to n-reinforcement, increasing the strength of a necessary argument increases the strength of the target, which obviously does not hold for the attack relation (see a-reinforcement).

The next principle says that if an initial weight of an argument decreases, its acceptability degree decreases as well (provided that it was not already zero).

Principle 15 (Proportionality) A semantics $\mathbf{S}$ satisfies proportionality iff, for any $W G N \mathbf{G}=\langle\mathcal{A}, w, \mathcal{R}, \mathcal{N}\rangle$ and $a, b \in$ $\mathcal{A}$ such that

$$
\begin{aligned}
& \text { - } \operatorname{Att}_{\mathbf{G}}(a)=\operatorname{Att}_{\mathbf{G}}(b), \\
& \text { - } \operatorname{Nec}_{\mathbf{G}}(a)=\operatorname{Nec}_{\mathbf{G}}(b), \\
& \text { - } w(a)>w(b) \text {, and } \\
& \text { - } \operatorname{Deg}_{\mathbf{G}} \mathbf{S}(a)>0 \text { or } \operatorname{Deg}_{\mathbf{G}} \mathbf{S}(b)>0,
\end{aligned}
$$

then $\operatorname{Deg}_{\mathbf{G}}^{\mathbf{S}}(a)>\operatorname{Deg}_{\mathbf{G}}^{\mathbf{S}}(b)$.

The following principle captures the main intuition behind the necessity relation: if the argument $b$ is necessary for $a$, then $a$ cannot be accepted if $b$ is not accepted as well. Therefore, the acceptability degree of $a$ is at most the acceptability degree of $b$.

Principle 16 (Trimming) A semantics $\mathbf{S}$ satisfies trimming iff, for any $W G N \mathbf{G}=\langle\mathcal{A}, w, \mathcal{R}, \mathcal{N}\rangle$ and $a, b \in \mathcal{A}$ such that $b \in \operatorname{Nec}_{\mathbf{G}}(a), \operatorname{Deg}_{\mathbf{G}}^{\mathbf{S}}(a) \leq \operatorname{Deg}_{\mathbf{G}}^{\mathbf{S}}(b)$.

Example 2 (Ex. 1 continued) Since $\operatorname{Deg}_{\mathrm{G}}^{\mathbf{S}}(x)=0.6$ and $\operatorname{Deg}_{\mathbf{G}}^{\mathbf{S}}(y)=0.8$, by trimming we have that $\operatorname{Deg}_{\mathbf{G}}^{\mathbf{S}}(a) \leq 0.6$ and $\operatorname{Deg}_{\mathbf{G}}^{\mathbf{S}}(b) \leq 0.8$. 
Another principle for ranking semantics is resilience [Amgoud et al., 2017], which says that an argument cannot lose all the intrinsic weight, i.e. that $w(a)>0$ implies $\operatorname{Deg}_{\mathbf{G}}^{\mathbf{S}}(a)>0$. This principle is not desirable in presence of necessities. Indeed, the main intuition behind the necessity relation is that an argument cannot be accepted if any of its necessary arguments is not accepted. This is formalized by the trimming principle, which implies that a necessary argument of degree 0 will always reduce the strength of its target to 0 , regardless of the initial weight of the target.

\section{Formal Analysis of the Principles}

In this proposition we show that if an argument is only attacked by arguments with a 0 acceptability value and the necessary arguments for it have an 1 acceptability value then its acceptability equals its basic strength for semantics satisfying independence, directionality, maximality, a-neutrality and nneutrality.

Proposition 1 Let a semantics $\mathbf{S}$ satisfy independence, directionality, maximality, a-neutrality and n-neutrality. Let $\mathbf{G}=\langle\mathcal{A}, w, \mathcal{R}, \mathcal{N}\rangle$ be $a W G N$ and $a \in \mathcal{A}$ an argument such that for every $x \in \operatorname{Att}_{\mathbf{G}}(a), \operatorname{Deg}_{\mathbf{G}} \mathbf{S}(x)=0$, and for every $y \in \operatorname{Nec}_{\mathbf{G}}(a), \operatorname{Deg}_{\mathbf{G}}^{\mathbf{S}}(y)=1$. Then, $\operatorname{Deg}_{\mathbf{G}}^{\mathbf{S}}(a)=w(a)$.

In the next proposition we show that if a semantics satisfy independence, directionality, maximality, a-weakening, n-weakening, a-neutrality and n-neutrality then its basic strength is an upper bound for its acceptability.

Proposition 2 Let a semantics $\mathbf{S}$ satisfy independence, directionality, maximality, a-weakening, $n$-weakening, a-neutrality and n-neutrality. Then, for every $W G N \mathbf{G}=\langle\mathcal{A}, w, \mathcal{R}, \mathcal{N}\rangle$ and every $a \in \mathcal{A}$ we have $\operatorname{Deg}_{\mathbf{G}} \mathbf{S}(a) \in[0, w(a)]$.

Arguments that have a basic strength equal to 0 , or more generally an acceptability degree 0 , cannot change their degrees if they become targets of additional attacks or necessities. This follows from directionality, independence, maximality, a-neutrality, n-neutrality, a-reinforcement and nreinforcement.

Proposition 3 Let a semantics $\mathbf{S}$ satisfy directionality, independence, maximality, a-neutrality, n-neutrality, areinforcement and $n$-reinforcement. Then, for every $W G N$ $\mathbf{G}=\langle\mathcal{A}, w, \mathcal{R}, \mathcal{N}\rangle$ and every $a, b, c \in \mathcal{A}$ such that $w(a)=$ $w(b), c \notin \operatorname{Att}_{\mathbf{G}}(a) \cup \operatorname{Nec}_{\mathbf{G}}(a)$ and

- $\operatorname{Nec}_{\mathbf{G}}(a)=\operatorname{Nec}_{\mathbf{G}}(b)$ and $\operatorname{Att}_{\mathbf{G}}(b)=\operatorname{Att}_{\mathbf{G}}(a) \cup\{c\}$, or

- $\operatorname{Att}_{\mathbf{G}}(a)=\operatorname{Att}_{\mathbf{G}}(b)$ and $\operatorname{Nec}_{\mathbf{G}}(b)=\operatorname{Nec}_{\mathbf{G}}(a) \cup\{c\}$, we have that $\operatorname{Deg}_{\mathbf{G}}^{\mathbf{S}}(a)=0$ implies $\operatorname{Deg}_{\mathbf{G}}^{\mathbf{S}}(b)=0$.

The monotony principle by Amgoud and Ben-Naim [2016] is stated for standard argumentation graphs (i.e. the graphs with attacks only) and says that a set $S$ of attackers weakens its target at least as much as any subset of $S$. The following results states that a generalization of that principle, that properly takes into account necessity relation of our framework, follows from a set of principles from Section 3 .
Proposition 4 Let a semantics $\mathbf{S}$ satisfy directionality, independence, maximality, a-neutrality, n-neutrality, $a$ reinforcement and $n$-reinforcement. Then, for every $W G N$ $\mathbf{G}=\langle\mathcal{A}, w, \mathcal{R}, \mathcal{N}\rangle$ and every $a, b \in \mathcal{A}$ if

- $w(a)=w(b)$

- $\operatorname{Att}_{\mathbf{G}}(a) \subseteq \operatorname{Att}_{\mathbf{G}}(b)$, and

- $\operatorname{Nec}_{\mathbf{G}}(a) \subseteq \operatorname{Nec}_{\mathbf{G}}(b)$

then $\operatorname{Deg}_{\mathbf{G}}^{\mathbf{S}}(a) \geq \operatorname{Deg}_{\mathbf{G}}^{\mathbf{S}}(b)$.

The following result shows that a set of our principles implies a property that reminds of counter-transitivity [Amgoud and Ben-Naim, 2013].

Proposition 5 Let a semantics $\mathbf{S}$ satisfy directionality, independence, maximality, equivalence, a-neutrality, n-neutrality, a-reinforcement and n-reinforcement. Then, for every WGN $\mathbf{G}=\langle\mathcal{A}, w, \mathcal{R}, \mathcal{N}\rangle$ and every $a, b \in \mathcal{A}$ if

- $w(a)=w(b)$ and

- there exist two injective functions $f: \operatorname{Att}(a) \rightarrow \operatorname{Att}(b)$ and $g: \operatorname{Nec}(a) \rightarrow \operatorname{Nec}(b)$ such that:

- for all $c \in \operatorname{Att}(a), \operatorname{Deg}_{\mathbf{G}}^{\mathbf{S}}(c) \leq \operatorname{Deg}_{\mathbf{G}}^{\mathbf{S}}(f(c))$

- for all $d \in \operatorname{Nec}(a) \operatorname{Deg}_{\mathbf{G}}^{\mathbf{S}}(d) \geq \operatorname{Deg}_{\mathbf{G}}^{\mathbf{S}}(g(d))$

then $\operatorname{Deg}_{\mathbf{G}}^{\mathbf{S}}(a) \geq \operatorname{Deg}_{\mathbf{G}}^{\mathbf{S}}(b)$.

The following two results give some dependencies between principles. By the following proposition, both a-counting and $\mathrm{n}$-counting follow from some other principles.

Proposition 6 Let $\mathbf{S}$ be a semantics which satisfies directionality, independence and maximality. Then

- If $\mathbf{S}$ satisfies a-neutrality and a-reinforcement, then $\mathbf{S}$ satisfies a-counting.

- If $\mathbf{S}$ satisfies n-neutrality and n-reinforcement, then $\mathbf{S}$ satisfies n-counting.

\section{Gradual Semantics for Argumentation Framework With Necessities}

In this section we provide the first gradual semantics for argumentation frameworks with necessities. As in case of any bipolar framework, it is hard to obtain a semantics that is well-defined for all graphs of the framework. The usual approach in the literature is to restrict the attention to the class of acyclic graphs [Amgoud and Ben-Naim, 2018; Rago et al., 2016]. The only exception is the work of Mossakowski and Neuhaus [2018]. However, that semantics is very simple and particular one, since it only takes into account the strongest attacker and the strongest supporter, while ignoring the values of other attackers and supporters. We will follow the standard approach and define semantics for several subclasses of graphs, including acyclic graphs. We consider the following three classes of graphs.

Notation: We denote by $\mathcal{G}_{a c}$ the set of all acyclic graphs (meaning that $\mathcal{R} \cup \mathcal{N}$ is aciclyc), by $\mathcal{G}_{\text {att }}$ the set of graphs with no necessities (i.e. where $\mathcal{N}=\emptyset$ ) and by $\mathcal{G}_{\text {nec }}$ the set of graphs with no attacks (i.e. $\mathcal{R}=\emptyset$ ). 
We use the probabilistic sum to aggregate the attackers, i.e. the commutative and associative function $x \oplus y=x+y-x y$. We define the semantics using the iterative method.

Definition 4 For a WGN $\mathbf{G}=\langle\mathcal{A}, w, \mathcal{R}, \mathcal{N}\rangle$ and $i \in$ $\{1,2,3, \ldots\}$ we define the function $f_{\mathbf{G}}^{i}$ inductively as follows:

$$
f_{\mathbf{G}}^{i}(a)=w(a) \frac{\prod_{x \in \operatorname{Nec}(a)} f_{\mathbf{G}}^{i-1}(x)}{e^{\bigoplus_{y \in \operatorname{Att}(a)} f_{\mathbf{G}}^{i-1}(y)}}
$$

with $f_{\mathbf{G}}^{0}(a)=w(a)$.

In the above definition we follow the usual convention that $\prod_{\alpha \in \emptyset} \alpha=1$ and $\bigoplus_{\alpha \in \emptyset} \alpha=0$.

The next result shows that $f_{\mathbf{G}}^{i}$ converges whenever $\mathbf{G}$ is acyclic, without attacks or without necessities.

Theorem 1 Let $\alpha \in\{a c, a t t, n e c\}$. Then, for every $\mathbf{G}=$ $\langle\mathcal{A}, w, \mathcal{R}, \mathcal{N}\rangle \in \mathcal{G}_{\alpha}$, for every $a \in \mathcal{A}, f_{\mathbf{G}}^{i}(a)$ converges when $i \rightarrow+\infty$.

This result allows us to define the semantics for the classes $\mathcal{G}_{a c}, \mathcal{G}_{a t t}$ and $\mathcal{G}_{n e c}$.

Definition 5 (Npe) The necessity product exponential semantics is a function Npe transforming any $W G N \mathbf{G}=$ $\langle\mathcal{A}, w, \mathcal{R}, \mathcal{N}\rangle \in \mathcal{G}_{\alpha}$, where $\alpha \in\{a c$, att, nec $\}$, into the function $\operatorname{Deg}_{\mathbf{G}}^{\text {Npe }}: \mathcal{A} \rightarrow[0,1]$, such that for any $a \in \mathcal{A}$,

$$
\operatorname{Deg}_{\mathbf{G}}^{\mathrm{Npe}}(a)=\lim _{i \rightarrow \infty} f_{\mathbf{G}}^{i}(a) \text {. }
$$

Example 3 (Ex. 1 continued) Let $\mathbf{G}$ be the WGN from Example 1. By applying Npe, we obtain $\operatorname{Deg}_{\mathbf{G}}^{\mathrm{Npe}}(x)=0.6$, $\operatorname{Deg}_{\mathbf{G}}^{\mathrm{Npe}}(y)=0.8, \operatorname{Deg}_{\mathbf{G}}^{\mathrm{Npe}}(c)=0.5, \operatorname{Deg}_{\mathbf{G}}^{\mathrm{Npe}}(a)=\frac{0.54}{\sqrt{e}} \approx 0.33$ and $\operatorname{Deg}_{\mathbf{G}}^{\mathrm{Npe}}(b)=\frac{0.72}{\sqrt{e}} \approx 0.44$.

Under Npe, the degree of every argument is bounded by its initial weight.

Proposition 7 For any $W G N \mathbf{G}=\langle\mathcal{A}, w, \mathcal{R}, \mathcal{N}\rangle \in \mathcal{G}_{\alpha}$, where $\alpha \in\{a c$, att, nec $\}$, for any $a \in \mathcal{A}$,

$$
\operatorname{Deg}_{\mathbf{G}}^{\mathrm{Npe}}(a) \in[0, w(a)] .
$$

We now prove that the Npe semantics can be represented by a set of equations relating the degree of an argument with the degrees of its attackers and necessities.

Theorem 2 Let $\alpha \in\{a c, a t t, n e c\}$. Then, for every $\mathbf{G}=$ $\langle\mathcal{A}, w, \mathcal{R}, \mathcal{N}\rangle \in \mathcal{G}_{\alpha}$, for every $a \in \mathcal{A}$

$$
\operatorname{Deg}_{\mathbf{G}}^{\mathrm{Npe}}(a)=w(a) \frac{\prod_{x \in \operatorname{Nec}(a)} \operatorname{Deg}_{\mathbf{G}}^{\mathrm{Npe}}(x)}{e^{\bigoplus_{y \in \operatorname{Att}(a)} \operatorname{Deg}_{\mathbf{G}}^{\mathrm{Npe}}(y)}} .
$$

The following theorem shows that for any graph from any of the considered three classes $\left(\mathcal{G}_{a c}, \mathcal{G}_{a t t}\right.$ and $\mathcal{G}_{\text {nec }}$ ), the set (one for each argument) of equations (1) has a unique solution.

\section{Theorem 3 Equation (1) uniquely characterizes Npe.}

Theorem 3 allows us to check the principle compliance. Our principles are properties of semantics that should hold for every graph. However, Npe is defined on three classes of graphs: $\mathcal{G}_{a c}, \mathcal{G}_{a t t}$ and $\mathcal{G}_{n e c}$, therefore it is only sensible to check the properties of Npe by restricting the principles to those classes. We next show that Npe satisfies all the principles.
Theorem 4 The Npe semantics satisfies all the principles on each of the three classes of graphs $\mathcal{G}_{a c}, \mathcal{G}_{\text {att }}$ and $\mathcal{G}_{\text {nec. }}$.

Finally, let us note that by applying the semantics Npe to the class $\mathcal{G}_{a t t}$, we obtain a novel semantics for all weighted argumentation graphs (not only for the acyclic ones). Similarly, restricting the Npe semantics to $\mathcal{G}_{n e c}$ leads to the first ranking-based semantics for weighted graphs with necessities only.

\section{Conclusions and Related Work}

In this paper, we study for the first time how to model necessities in ranking based semantics. This allows us to combine two things both needed in order to model certain practical scenarios:

- First, the ability of ranking based semantics to model the attacks that only weaken their targets (and not completely destroy them).

- Second, the use of necessity relation in order to model the fact that an argument $a$ is necessary for the argument $b$ (i.e. the rejection of $a$ should lead to complete rejection of $b$ ).

As explained in the paper and made intuitively obvious by our motivating example in the introduction, necessity does not behave as a type of support, nor a type of attack. The study of its behaviour for ranking semantics allowed us to basically identify a new type of relation. To this end necessity shares the property of attack relations in weakening their targets, but also an important property of support in the fact that the stronger the source, the stronger the target.

Placing our work within the relevant literature is thus a difficult exercise because none of the approaches identified the specificities of the necessity relations as explained above. However, we would like to highlight in chronological order five important papers that should be mentioned at this point. First we mention the noteworthy work of Cayrol and Lagasquie-Schiex [2005a], who were the first to propose to rank arguments in bipolar argumentation frameworks by aggregating the values of all parents of arguments. Rago et al. [2016] introduce DF-QuAD to deal with quantitative argumentation debates in order to allow for automated decision support, whereas Amgoud and Ben-Naim [2018] provide a comprehensive and inspiring study of ranking semantics and their principles for bipolar argumentation systems when considering a general approach to support. The work of Mossakowski and Neuhaus [2018] is an important indepth analysis of the technicalities behind convergence results in bipolar argumentation frameworks with a particular emphasis on the underlying graph structure of the argumentation framework. Finally, let us also mention the work by Potyka [2019], which generalizes the convergence conditions introduced by Mossakowski and Neuhaus.

\section{References}

[Amgoud and Ben-Naim, 2013] Leila Amgoud and Jonathan Ben-Naim. Ranking-based semantics for argumentation frameworks. In International Conference 
on Scalable Uncertainty Management, pages 134-147. Springer, 2013.

[Amgoud and Ben-Naim, 2016] Leila Amgoud and Jonathan Ben-Naim. Axiomatic foundations of acceptability semantics. In International Conference on Principles of Knowledge Representation and Reasoning, pages 2-11, 2016.

[Amgoud and Ben-Naim, 2018] Leila Amgoud and Jonathan Ben-Naim. Weighted bipolar argumentation graphs: Axioms and semantics. In International Joint Conference on Artificial Intelligence, pages 5194-5198, 2018.

[Amgoud and Doder, 2018] Leila Amgoud and Dragan Doder. Gradual semantics for weighted graphs: An unifying approach. In International Conference on Principles of Knowledge Representation and Reasoning, pages 613614, 2018.

[Amgoud and Doder, 2019] Leila Amgoud and Dragan Doder. Gradual semantics accounting for varied-strength attacks. In International Conference on Autonomous Agents and MultiAgent Systems, pages 1270-1278, 2019.

[Amgoud et al., 2008] Leila Amgoud, Claudette Cayrol, Marie-Christine Lagasquie-Schiex, and Pierre Livet. On bipolarity in argumentation frameworks. International Journal of Intelligent Systems, 23(10):1062-1093, 2008.

[Amgoud et al., 2017] Leila Amgoud, Jonathan Ben-Naim, Dragan Doder, and Srdjan Vesic. Acceptability semantics for weighted argumentation frameworks. In International Joint Conference on Artificial Intelligence, pages 56-62, 2017.

[Boella et al., 2010] Guido Boella, Dov M Gabbay, Leon van der Torre, and Serena Villata. Support in abstract argumentation. In International Conference on Computational Models of Argument, pages 40-51, 2010.

[Bonzon et al., 2016] Elise Bonzon, Jérôme Delobelle, Sébastien Konieczny, and Nicolas Maudet. A comparative study of ranking-based semantics for abstract argumentation. In AAAI Conference on Artificial Intelligence, pages 914-920, 2016.

[Boudhar et al., 2012] Imane Boudhar, Farid Nouioua, and Vincent Risch. Handling preferences in argumentation frameworks with necessities. In International Conference on Agents and Artificial Intelligence, pages 340-345, 2012.

[Cayrol and Lagasquie-Schiex, 2005a] Claudette Cayrol and Marie-Christine Lagasquie-Schiex. Gradual valuation for bipolar argumentation frameworks. In European Conference on Symbolic and Quantitative Approaches to Reasoning and Uncertainty, pages 366-377. Springer, 2005.

[Cayrol and Lagasquie-Schiex, 2005b] Claudette Cayrol and Marie-Christine Lagasquie-Schiex. On the acceptability of arguments in bipolar argumentation frameworks. In European Conference on Symbolic and Quantitative Approaches to Reasoning and Uncertainty, pages 378-389. Springer, 2005.
[Cayrol and Lagasquie-Schiex, 2013] Claudette Cayrol and Marie-Christine Lagasquie-Schiex. Bipolarity in argumentation graphs: Towards a better understanding. International Journal of Approximate Reasoning, 54(7):876899, 2013.

[Cohen et al., 2015] Andrea Cohen, Sebastian Gottifredi, Alejandro Javier Garcia, and Guillermo Ricardo Simari. An approach to abstract argumentation with recursive attack and support. J. Applied Logic, 13(4):509-533, 2015.

[Dung, 1995] Phan Minh Dung. An argumentation-theoretic foundation for logic programming. The Journal of logic programming, 22(2):151-177, 1995.

[Gottifredi et al., 2018] Sebastian Gottifredi, Andrea Cohen, Alejandro J Garcia, and Guillermo R Simari. Characterizing acceptability semantics of argumentation frameworks with recursive attack and support relations. Artificial Intelligence, 262:336-368, 2018.

[Leite and Martins, 2011] João Leite and João G. Martins. Social abstract argumentation. In International Joint Conference on Artificial Intelligence, pages 2287-2292, 2011.

[Matt and Toni, 2008] Paul-Amaury Matt and Francesca Toni. A game-theoretic measure of argument strength for abstract argumentation. In European Workshop on Logics in Artificial Intelligence, pages 285-297. Springer, 2008.

[Mossakowski and Neuhaus, 2018] Till Mossakowski and Fabian Neuhaus. Modular semantics and characteristics for bipolar weighted argumentation graphs. CoRR, abs/1807.06685, 2018.

[Nouioua and Risch, 2011] Farid Nouioua and Vincent Risch. Argumentation frameworks with necessities. In International Conference on Scalable Uncertainty Management, pages 163-176. Springer, 2011.

[Nouioua, 2013] Farid Nouioua. AFs with necessities: further semantics and labelling characterization. In International Conference on Scalable Uncertainty Management, pages 120-133. Springer, 2013.

[Oren and Norman, 2008] Nir Oren and Timothy J Norman. Semantics for evidence-based argumentation. In International Conference on Computational Models of Argument, pages 276-284. IOS Press, 2008.

[Potyka, 2019] Nico Potyka. Extending modular semantics for bipolar weighted argumentation. In International Conference on Autonomous Agents and MultiAgent Systems, pages 1722-1730, 2019.

[Rago et al., 2016] Antonio Rago, Francesca Toni, Marco Aurisicchio, and Pietro Baroni. Discontinuity-free decision support with quantitative argumentation debates. In International Conference on Principles of Knowledge Representation and Reasoning, pages 63-73, 2016.

[Villata et al., 2012] Serena Villata, Guido Boella, Dov M Gabbay, and Leendert Van Der Torre. Modelling defeasible and prioritized support in bipolar argumentation. Annals of Mathematics and Artificial Intelligence, 66(14):163-197, 2012. 